\title{
Interacciones de la DL-Alanina en Soluciones Acuosas de Trifluorometanosulfonato de 1-Butil, 3-Metilimidazolio a Diferentes Temperaturas
}

\author{
Manuel S. Páez ${ }^{(1) \star}$, María K. Vergara(1), Nicolás A. De la Espriella ${ }^{(2)}$. \\ (1) Universidad de Córdoba, Departamento de Química, Facultad de Ciencias Básicas, Carrera 6 No. 76 - \\ 103. Km 3. vía Cereté. Córdoba. Colombia. (e-mail: mspaezm@unal.edu.co.) \\ (2) Universidad de Córdoba. Departamento de Física. Facultad de Ciencias Básicas. Grupo GAMASCO. \\ Carrera 6 No. 76-103. Km 3. vía Cereté. Córdoba. Colombia.
}

${ }^{*}$ Autor a quien debe dirigirse la correspondencia.

Recibido Jun. 2, 2014; Aceptado Ago. 8, 2014; Versión final recibida Sep. 8, 2014

\begin{abstract}
Resumen
Se determinó la densidad de DL-alanina en soluciones acuosas del líquido iónico trifluorometanosulfonato de 1-Butil-3-metil imidazolio en el intervalo de temperaturas de 283.15 a $313.15 \mathrm{~K}$ utilizando un densímetro de tubo vibratorio Anton Paar DMA 5000. Se calcularon los volúmenes molares aparentes, volúmenes molares aparentes a dilución infinita, la segunda derivada del volumen molar parcial a dilución infinita con respecto a la temperatura, el volumen molar parcial de transferencia a dilución infinita y los números de hidratación. Se encontró que los valores de la segunda derivada del volumen molar aparente límite con temperatura son negativos a todas las concentraciones del solvente mixto. Esto indica que se favorecen las interacciones soluto-solvente y que la DL-alanina actúa como un desestabilizador de la estructura del solvente.
\end{abstract}

Palabras clave: propiedades volumétricas, volúmenes molares aparentes, DL-alanina en soluciones acuosas del líquido iónico

\section{Interactions of DL-Alanine in Aqueous Solutions of 1-Butyl, 3- Methylimidazolium Trifluoromethanesulfonate at Different Temperatures}

\begin{abstract}
Densities of DL-alanine in aqueous solutions of ionic liquid 1-butyl-3- methylimidazolium trifluoromethanesulfonate were determined at temperatures ranging from 283.15 to $313.15 \mathrm{~K}$ using an Anton Paar DMA 5000 vibrating tube densitometer. The apparent molar volume, the infinite dilution apparent molar volume, the second derivative of the infinite dilution partial molar volume with respect to temperature, the partial molar volume of transfer at infinite dilution, and the number of hydration were calculated. It was found that the values of the second derivative of the limiting apparent molar volume with temperature are negative at all concentrations of the mixed solvent. This indicates that solute-solvent interactions are favored and that the DL-alanine acts as a disruptor of the structure of the solvent.
\end{abstract}

Keywords: volumetric properties; apparent molar volume; DL-alanine in aqueous ionic liquid. 


\section{INTRODUCCIÓN}

Los aminoácidos como unidades estructurales fundamentales de péptidos y proteínas juegan un importante papel en los sistemas biológicos, porque afectan la solubilidad, la desnaturalización y la actividad de biomoléculas. El estudio de los efectos de las propiedades termofísicas de estos compuestos modelos en soluciones electrolíticas proporciona información importante acerca de las interacciones soluto-solvente y soluto-soluto en biomoléculas (Shekaari et al., 2010).

Los líquidos iónicos a temperatura ambiente son una clase de sales orgánicas que se componen enteramente de iones y son líquidos en condiciones alrededor de la temperatura ambiente en su estado puro (Páez et al., 2013; Cortés et al., 2010). El estudio de estos líquidos en solución puede ayudar a entender el efecto que tienen las interacciones electrostáticas e hidrofóbicas sobre la estabilidad de los aminoácidos; Así mismo, es de esperar, que estos compuestos tengan influencia sobre la conformación macromolecular de las biomoléculas, mediante el debilitamiento de las interacciones repulsivas o atractivas inter o intra cadena y carga-carga y afectando las interacciones hidrofóbicas de sus cadenas laterales con los grupos alquílicos. Pese a los grandes esfuerzos para comprender esta temática de estudio, el comportamiento de este tipo particular de sistemas aun no ha sido del todo bien entendido (Páez et al., 2014).

En este trabajo se estudian, las propiedades volumétricas, debido a que estas son útiles para determinar el efecto que causa la adición de una sal sobre el comportamiento de los aminoácidos; siendo esta información de gran importancia para el estudio de la hidratación de péptidos y proteínas en medios salinos (Deeying et al., 2009; Páez et al., 2013). Por tal motivo, aquí se reportan las densidades $\rho /\left(\mathrm{g} / \mathrm{cm}^{3}\right)$ de DLalanina en función de la molalidad del líquido iónico [BMIm][OTF] a la presión de 1.011 bar, y a las temperaturas de $(283.15 ; 288.15 ; 293.15 ; 298.15,303.15 ; 308.15$ y 313,15$) \mathrm{K}$. Los valores experimentales de $\rho$ se usaron para calcular los volúmenes molares aparentes $V_{\phi} /\left(\mathrm{cm}^{3} \cdot \mathrm{mol}^{-1}\right)$, los volúmenes molares aparentes a dilución infinita $V_{\phi}^{o} /\left(\mathrm{cm}^{3} \cdot \mathrm{mol}^{-1}\right)$, la pendiente límite experimental $\mathrm{S}_{\mathrm{v}} /\left(\mathrm{m}^{3} \cdot \mathrm{mol}^{-2} \cdot \mathrm{Kg}\right)$, la segunda derivada del volumen molar aparente límite con temperatura $\left(\partial^{2} \mathrm{~V}_{\phi}^{0} / \partial \mathrm{T}^{2}\right) /\left(\mathrm{mol} . \mathrm{K}^{-1}\right)$, los volúmenes molares de transferencia $\Delta V_{\phi}^{\mathrm{o}} /\left(\mathrm{cm}^{3}\right.$. $\left.\mathrm{mol}^{-1}\right)$, y los números de hidratación, $\mathrm{N}_{\mathrm{H}}$. El comportamiento de estos parámetros con la concentración y la temperatura fueron analizados en términos de las interacciones que ocurren a nivel de la solución.

\section{MATERIALES Y MÉTODOS}

Se empleó DL-alanina grado analítico (99\%) adquirida de la casa comercial Alfa Aesar y líquido iónico [BMIm][OTF] obtenido de Across Organic con una pureza $\geq 99 \%$. Antes de su uso la DL-alanina fue recristalizada en soluciones acuosas de etanol y secada al vacío sobre $\mathrm{P}_{2} \mathrm{O}_{5}$ (Armarego, 2009). El contenido de agua de este LI se determinó usando el método de Karl Fischer. El agua usada para preparar las soluciones fue bidestilada y desgasificada y presentó una conductividad menor que $2 \mu \mathrm{S}$. Las soluciones fueron preparadas en la escala de molalidad utilizando el método gravimétrico, en recipientes de vidrio con tapa, tomando todas las precauciones necesarias para evitar la contaminación de las muestras y la pérdida de masa por evaporación de los líquidos utilizados. Todas las medidas de masa fueron realizadas en una balanza analítica Ohaus con una incertidumbre de $\pm 1 \times 10^{-4} \mathrm{~g}$. Las densidades de los componentes puros y sus mezclas fueron determinadas en un densímetro de tubo vibratorio Anton Paar DMA 5000, con una incertidumbre de $1 \times 10^{-5} \mathrm{~g} / \mathrm{cm}^{3}$ y un control de temperatura de $\pm 0.001 \mathrm{~K}$ en el intervalo de temperatura (283.15-313.15) K.

\section{RESULTADOS Y DISCUSIÓN}

Los datos experimentales de densidad, de los distintos sistemas seudobinarios derivados del [BMIm][OTF] fueron ajustados por un procedimiento de mínimos cuadrados usando la ecuación

$\rho=a+b m+c T+d m^{2}+e T^{2}+f m T+g m^{3}+h T^{3}+i m T^{2}+j m^{2} T$

Donde $\rho$ es la densidad de la solución, $m$ es la molalidad de la D-L alanina en la mezcla [BMIm][OTF] + agua, T es la temperatura absoluta, $\sigma$ es la desviación estándar y las letras minúsculas desde $a$ hasta $j$ son parámetros ajustables. Los resultados de este ajuste se muestran en la Tabla 1 y ellos permiten obtener densidades con la desviación estándar especificada en dicha tabla.

Los valores de los volúmenes molares aparentes fueron calculados de los valores de densidad generados a partir de la ecuación (1) con ayuda de la ecuación (2) (Páez et al., 2013): 
$V_{\phi}=\frac{M}{\rho}-\frac{1000\left(\rho-\rho_{0}\right)}{m \rho \rho_{0}}$

Donde $\mathrm{M}$ es la masa molar del aminoácido, $\mathrm{m}$ es la molalidad de la DL-alanina, que es definida como las moles de DL-alanina por kilogramo de solvente (solución acuosa de [BMIm][OTF] ), $\rho$ y $\rho_{0}$ son respectivamente la densidad de la solución (DL-alanina + solución acuosa de [BMIm][OTF]) y la densidad del solvente (solución acuosa de [BMIm][OTF]). La incertidumbre para los valores de $\mathrm{V}_{\phi}$ fue menor que 0.04 $\mathrm{cm}^{3} \mathrm{~mol}^{-1}$. Finalmente se obtuvieron los volúmenes molares aparentes a dilución infinita $\mathrm{V}_{\phi}^{\mathrm{o}}$ usando un procedimiento de regresión lineal con ayuda de la ecuación (3):

$V_{\phi}=V_{\phi}^{o}+S_{v} m$

Tabla 1. Parámetros de ajuste de la ecuación (1), para las mezclas seudobinarias derivadas de las soluciones acuosas de líquido iónico [BMIm][OTF], válida en el intervalo de (0.0000-1.0980)maa y a las temperaturas de $(283.15,288.15,293.15,298.15,303.15,308.15$ y 313.15$) \mathrm{K}$.

\begin{tabular}{ccccccc}
\hline $\mathrm{mLI}\left(\mathrm{mol} \cdot \mathrm{Kg}^{-1}\right)$ & $0.0500 \mathrm{~m}$ & $0.0800 \mathrm{~m}$ & $0.2000 \mathrm{~m}$ & $0.4000 \mathrm{~m}$ & $0.8000 \mathrm{~m}$ & $1.0000 \mathrm{~m}$ \\
\hline \multicolumn{7}{c}{ Parámetros de ajuste de la ecuación 1} \\
\hline$a$ & $-7.0285 \mathrm{E}-01$ & $-2.3243 \mathrm{E}-01$ & $2.7721 \mathrm{E}-01$ & $1.5942 \mathrm{E}-01$ & $8.7094 \mathrm{E}-01$ & $1.8348 \mathrm{E}+00$ \\
$b$ & $3.0775 \mathrm{E}-01$ & $5.4955 \mathrm{E}-02$ & $7.5603 \mathrm{E}-02$ & $5.4133 \mathrm{E}-02$ & $1.2644 \mathrm{E}-01$ & $7.5090 \mathrm{E}-02$ \\
$c$ & $1.6189 \mathrm{E}-02$ & $1.1506 \mathrm{E}-02$ & $6.5766 \mathrm{E}-03$ & $8.0314 \mathrm{E}-03$ & $1.2894 \mathrm{E}-03$ & $-8.2222 \mathrm{E}-03$ \\
$d$ & $-4.5097 \mathrm{E}-02$ & $-1.0774 \mathrm{E}-02$ & $-1.3787 \mathrm{E}-02$ & $-1.6729 \mathrm{E}-02$ & $-1.2485 \mathrm{E}-02$ & $-9.7999 \mathrm{E}-03$ \\
$e$ & $-5.0107 \mathrm{E}-05$ & $-3.4665 \mathrm{E}-05$ & $-1.8366 \mathrm{E}-05$ & $-2.3603 \mathrm{E}-05$ & $-1.4624 \mathrm{E}-06$ & $3.0207 \mathrm{E}-05$ \\
$f$ & $-1.7769 \mathrm{E}-03$ & $-1.2364 \mathrm{E}-04$ & $-2.6423 \mathrm{E}-04$ & $-1.1599 \mathrm{E}-04$ & $-6.0783 \mathrm{E}-04$ & $-2.6506 \mathrm{E}-04$ \\
$g$ & $1.0039 \mathrm{E}-03$ & $6.5376 \mathrm{E}-05$ & $-1.9755 \mathrm{E}-04$ & $1.7383 \mathrm{E}-07$ & $1.3018 \mathrm{E}-05$ & $-2.5602 \mathrm{E}-05$ \\
$h$ & $5.0296 \mathrm{E}-08$ & $3.3354 \mathrm{E}-08$ & $1.5288 \mathrm{E}-08$ & $2.1395 \mathrm{E}-08$ & $-3.0468 \mathrm{E}-09$ & $-3.8267 \mathrm{E}-08$ \\
$i$ & $2.8241 \mathrm{E}-06$ & $1.2528 \mathrm{E}-07$ & $3.6202 \mathrm{E}-07$ & $1.0476 \mathrm{E}-07$ & $9.3272 \mathrm{E}-07$ & $3.5798 \mathrm{E}-07$ \\
$j$ & $1.4154 \mathrm{E}-04$ & $2.6985 \mathrm{E}-05$ & $3.9091 \mathrm{E}-05$ & $4.7665 \mathrm{E}-05$ & $3.3729 \mathrm{E}-05$ & $2.4872 \mathrm{E}-05$ \\
$\sigma$ & $2.2060 \mathrm{E}-05$ & $1.4870 \mathrm{E}-05$ & $4.6830 \mathrm{E}-05$ & $3.8300 \mathrm{E}-05$ & $2.6870 \mathrm{E}-05$ & $4.7710 \mathrm{E}-05$ \\
\hline
\end{tabular}

Donde $\mathrm{S}_{\mathrm{v}}$ es la pendiente límite experimental, también considerada como el coeficiente volumétrico virial, el cual caracteriza las interacciones pares entre las especies de soluto solvatados en la solución, mientras que El estudio del volumen molar aparente límite $V_{\phi}^{o}$ de un soluto, provee información importante acerca de los procesos de hidratación que le están asociados. Además, el estudio de la dependencia con la concentración del volumen molar parcial y del volumen molar aparente, puede ser utilizado para analizar las interacciones entre los solutos (Millero., 1978; Shekaari et al., 2010).

Los valores de $V_{\phi}^{o}\left(\mathrm{~cm}^{3} \mathrm{~mol}^{-1}\right)$ y $\mathrm{S}_{\mathrm{v}}$ se muestran en la Tabla 2. Valores positivos de $\mathrm{S}_{\mathrm{v}}$ para las mezclas de DL-alanina sugieren que el coeficiente volumétrico virial par es dominado por las interacciones de los grupos funcionales cargados del aminoácido con los iones del líquido iónico. Los valores de $\mathrm{V}_{\phi}^{\circ}$ son positivos para todas las temperaturas y concentraciones del solvente mixto, además disminuyen con el incremento de la concentración del [BMIm][OTF] a todas las temperaturas, lo cual podría ser interpretado como una contracción del volumen a dilución infinita debido al incremento de las interacciones soluto-solvente. Así mismo, los valores de $V_{\phi}^{o}$ incrementan sistemáticamente con el aumento de temperatura. Los valores experimentales de $V_{\phi}^{o}$ fueron correlacionados con temperatura usando la ecuación (4):

$\mathrm{V}_{\phi}^{0}=\mathrm{A}+\mathrm{BT}+\mathrm{CT}^{2}$

Donde $\mathrm{A}$, B y $\mathrm{C}$ son constantes y $\mathrm{T}$ es la temperatura absoluta. Los valores de $\left(\partial^{2} \mathrm{~V}_{\phi}^{0} / \partial \mathrm{T}^{2}\right)$, son negativos (desviación estándar de 0.0039), para todas las concentraciones del solvente mixto, lo cual sugiere de acuerdo con Hepler (Hepler, 1969), que la DL-alanina se comporta como un soluto disruptor de la estructura del solvente en soluciones acuosas de [BMIm][OTF].Los valores se presentan en la Tabla 3. 
Es importante destacar que para investigar el efecto que causa un soluto sobre la estructura del solvente, diversos investigadores han usado el criterio de Hepler (Makhatadze et al., 1962; Sharp et al., 1995; Kumar, 2008).

Tabla 2: Volumenes molares aparentes a dilucion infinita para la DL-alanina en soluciones acuosas de [BMIm][OTF] a las temperaturas de 283.15, 288.15, $293.15,298.15,303.15,308.15$ y $313.15 \mathrm{~K}$.

\begin{tabular}{|c|c|c|c|c|c|c|c|}
\hline $\mathrm{T}$ & $/ \mathrm{K}$ & \multicolumn{6}{|c|}{283.15} \\
\hline $\mathrm{m}[\mathrm{BMIm}][\mathrm{OTF}]$ & $/\left(\mathrm{mol} . \mathrm{Kg}^{-1}\right)$ & 0.0500 & 0.0800 & 0.2000 & 0.4000 & 0.8000 & 1.0000 \\
\hline $\mathrm{V}_{\phi}^{\mathrm{o}}$ & $/\left(\mathrm{cm}^{3} \mathrm{~mol}^{-1}\right)$ & 58.90 & 58.82 & 58.73 & 58.60 & 58.40 & 58.32 \\
\hline Sv & $/\left(\mathrm{m}^{3} \cdot \mathrm{mol}^{-2} \cdot \mathrm{Kg}\right)$ & 1.26 & 1.24 & 1.12 & 1.12 & 0.92 & 0.94 \\
\hline $\mathrm{R}^{2}$ & & 0.9994 & 0.9992 & 0.9997 & 0.9990 & 0.9985 & 0.9999 \\
\hline $\mathrm{T}$ & $/ \mathrm{K}$ & \multicolumn{6}{|c|}{288.15} \\
\hline $\mathrm{V}_{\phi}^{\mathrm{o}}$ & $/\left(\mathrm{cm}^{3} \mathrm{~mol}^{-1}\right)$ & 59.36 & 59.20 & 59.16 & 59.07 & 58.91 & 58.82 \\
\hline Sv & $/\left(\mathrm{m}^{3} \cdot \mathrm{mol}^{-2} \cdot \mathrm{Kg}\right)$ & 1.26 & 1.24 & 1.12 & 1.12 & 0.92 & 0.94 \\
\hline $\mathrm{R}^{2}$ & & 0.9994 & 0.9992 & 0.9997 & 0.9990 & 0.9985 & 0.9999 \\
\hline $\mathrm{T}$ & $/ \mathrm{K}$ & \multicolumn{6}{|c|}{293.15} \\
\hline $\mathrm{V}_{\phi}^{\mathrm{o}}$ & $/\left(\mathrm{cm}^{3} \cdot \mathrm{mol}^{-1}\right)$ & 59.64 & 59.53 & 59.40 & 59.34 & 59.22 & 59.19 \\
\hline Sv & $/\left(\mathrm{m}^{3} \cdot \mathrm{mol}^{-2} \cdot \mathrm{Kg}\right)$ & 1.12 & 0.98 & 0.98 & 0.96 & 0.92 & 0.85 \\
\hline $\mathrm{R}^{2}$ & & 0.9993 & 0.9991 & 0.9935 & 0.9997 & 0.9995 & 0.9990 \\
\hline $\mathrm{T}$ & $/ \mathrm{K}$ & \multicolumn{6}{|c|}{298.15} \\
\hline $\mathrm{V}_{\phi}^{\mathrm{o}}$ & $/\left(\mathrm{cm}^{3} \cdot \mathrm{mol}^{-1}\right)$ & 59.96 & 59.84 & 59.75 & 59.67 & 59.54 & 59.56 \\
\hline Sv & $/\left(\mathrm{m}^{3} \cdot \mathrm{mol}^{-2} \cdot \mathrm{Kg}\right)$ & 0.93 & 0.69 & 0.69 & 0.70 & 0.75 & 0.58 \\
\hline $\mathrm{R}^{2}$ & & 0.9993 & 0.9990 & 0.9991 & 0.9996 & 0.9996 & 0.9991 \\
\hline$T$ & $/ \mathrm{K}$ & \multicolumn{6}{|c|}{303.15} \\
\hline $\mathrm{V}_{\phi}^{\mathrm{o}}$ & $/\left(\mathrm{cm}^{3} \mathrm{~mol}^{-1}\right)$ & 60.22 & 60.06 & 60.00 & 59.91 & 59.83 & 59.76 \\
\hline Sv & $/\left(\mathrm{m}^{3} \cdot \mathrm{mol}^{-2} \cdot \mathrm{Kg}\right)$ & 0.88 & 0.56 & 0.48 & 0.56 & 0.54 & 0.54 \\
\hline $\mathrm{R}^{2}$ & & 0.9993 & 0.9994 & 0.9975 & 0.9990 & 0.9994 & 0.9995 \\
\hline $\mathrm{T}$ & $/ \mathrm{K}$ & \multicolumn{6}{|c|}{308.15} \\
\hline $\mathrm{V}_{\phi}^{\mathrm{o}}$ & $/\left(\mathrm{cm}^{3} \cdot \mathrm{mol}^{-1}\right)$ & 60.42 & 60.25 & 60.21 & 60.17 & 60.11 & 60.02 \\
\hline Sv & $/\left(\mathrm{m}^{3} \cdot \mathrm{mol}^{-2} \cdot \mathrm{Kg}\right)$ & 0.81 & 0.50 & 0.38 & 0.36 & 0.35 & 0.34 \\
\hline $\mathrm{R}^{2}$ & & 0.9996 & 0.9997 & 0.9995 & 0.9986 & 0.9996 & 0.9995 \\
\hline$T$ & $/ \mathrm{K}$ & \multicolumn{6}{|c|}{313.15} \\
\hline $\mathrm{V}_{\phi}^{\mathrm{o}}$ & $/\left(\mathrm{cm}^{3} \mathrm{~mol}^{-1}\right)$ & 60.69 & 60.39 & 60.34 & 60.30 & 60.22 & 60.15 \\
\hline Sv & $/\left(\mathrm{m}^{3} \cdot \mathrm{mol}^{-2} \cdot \mathrm{Kg}\right)$ & 0.71 & 0.50 & 0.39 & 0.36 & 0.37 & 0.70 \\
\hline $\mathrm{R}^{2}$ & & 0.9991 & 0.9995 & 0.9980 & 0.9987 & 0.9996 & 0.9991 \\
\hline
\end{tabular}

Tabla 3: Valores de $\left(\partial^{2} \mathrm{~V}_{\phi}^{0} / \partial \mathrm{T}^{2}\right)$ de la ecuación (4) para el aminoácido DL-alanina, en soluciones acuosas de [BMIm][OTF]

\begin{tabular}{lcccccc}
\hline m[BMlm][OTF] $\left(\mathrm{mol}^{\mathrm{K}} \mathrm{Kg}^{-1}\right)$ & 0.0500 & 0.0800 & 0.2000 & 0.4000 & 0.8000 & 10000 \\
\hline$\left(\partial^{2} \mathrm{~V}_{\phi}^{0} / \partial \mathrm{T}^{2}\right) 10^{2} \mathrm{molK}^{-1}$ & -3.7 & -4.7 & -4.3 & -4.5 & -5.1 & -5.8 \\
\hline
\end{tabular}


Los volúmenes molares de transferencia a dilución infinita $\Delta \mathrm{V}_{\phi}^{o}$ de la $\mathrm{DL}$-alanina desde el agua pura hasta las soluciones de [BMIm][OTF] fueron obtenidos utilizando la ecuación (5):

$\Delta \mathrm{V}_{\phi}^{\mathrm{o}}=\mathrm{V}_{\phi}^{\mathrm{o}}$ (en solución acuosa de [BMIm][OTF] $]$ ) $-\mathrm{V}_{\phi}^{\mathrm{o}}$ (en agua)

Las cantidades correspondiente para el volumen molar aparente límite de la DL-alanina en medio acuoso se presentan en la tabla 4.

Tabla 4: Volumenes molares aparentes a dilucion infinita para la DL-alanina en agua a las temperaturas de 283.15 , $288.15,293,15,298.15,303.15,308.15$ y $313.15 \mathrm{~K}$.

\begin{tabular}{cc}
\hline $\mathrm{T} / \mathrm{K}$ & $\mathrm{V}_{\phi}^{\mathrm{o}} /\left(\mathrm{cm}^{3} \cdot \mathrm{mol}^{-1}\right)$ \\
\hline 283.15 & 59.07 \\
288.15 & 59.62 \\
293.15 & 60.04 \\
298.15 & 60.25 \\
303.15 & 60.56 \\
308.15 & 60.92 \\
313.15 & 61.20 \\
\hline
\end{tabular}

Estos valores son utilizados para realizar el cálculo de los volúmenes molares de transferencia a dilución infinita presentados en la Tabla 5 , donde se observa que los valores obtenidos son negativos a todas las temperaturas. De igual manera, los valores de $\Delta \mathrm{V}_{\phi}^{o}$ disminuyen con el incremento de la concentración de [BMIm][OTF] en las mezclas. A dilución infinita, donde no existen las interacciones soluto-soluto, el volumen de transferencia proporciona información acerca de la interacción de un co-solvente y un soluto. (Pal et al., 2011; Páez et al., 2013)

Tabla 5: Volumenes molares de transferencia $\Delta \mathbf{V}_{\phi}^{\mathbf{o}}$, para la DL-alanina en soluciones acuosas de [BMIm][OTF] a las temperaturas de 283.15, 288.15, 293,15, 298.15, 303.15, 308.15 y $313.15 \mathrm{~K}$.

\begin{tabular}{ccccccc}
\hline & \multicolumn{5}{c}{$\mathrm{m}[\mathrm{BMIm}][\mathrm{OTF}]\left(\mathrm{mol}_{\mathrm{Kg}}{ }^{-1}\right)$} \\
\cline { 2 - 6 }$. \mathrm{T} / \mathrm{K}$ & 0.0500 & 0.0800 & 0.2000 & 0.4000 & 0.8000 & 1.0000 \\
\hline 283.15 & $-0.17 \pm 0.02$ & $-0.25 \pm 0.01$ & $-0.34 \pm 0.01$ & $-0.47 \pm 0.02$ & $-0.67 \pm 0.02$ & $-0.75 \pm 0.01$ \\
288.15 & $-0.26 \pm 0.02$ & $-0.42 \pm 0.02$ & $-0.46 \pm 0.01$ & $-0.55 \pm 0.02$ & $-0.71 \pm 0.02$ & $-0.80 \pm 0.02$ \\
293.15 & $-0.40 \pm 0.01$ & $-0.51 \pm 0.01$ & $-0.64 \pm 0.01$ & $-0.70 \pm 0.01$ & $-0.82 \pm 0.02$ & $-0.85 \pm 0.01$ \\
298.15 & $-0.29 \pm 0.02$ & $-0.41 \pm 0.01$ & $-0.50 \pm 0.02$ & $-0.58 \pm 0.02$ & $-0.71 \pm 0.02$ & $-0.72 \pm 0.02$ \\
303.15 & $-0.34 \pm 0.01$ & $-0.50 \pm 0.02$ & $-0.55 \pm 0.01$ & $-0.64 \pm 0.02$ & $-0.73 \pm 0.01$ & $-0.80 \pm 0.01$ \\
308.15 & $-0.50 \pm 0.02$ & $-0.67 \pm 0.01$ & $-0.71 \pm 0.01$ & $-0.75 \pm 0.01$ & $-0.82 \pm 0.02$ & $-0.90 \pm 0.02$ \\
313.15 & $-0.51 \pm 0.02$ & $-0.81 \pm 0.01$ & $-0.86 \pm 0.02$ & $-0.90 \pm 0.02$ & $-0.98 \pm 0.01$ & $-1.05 \pm 0.01$ \\
\hline
\end{tabular}

Siguiendo el modelo de las coesferas solapadas de Frank y Evans, (Liu, 2010) y en virtud, a que en una solución de un aminoácido en mezclas acuosas de un líquido iónico se pueden presentar interacciones (Singh et al., 2007): I. Ion-ion entre el anión del líquido iónico en nuestro caso el grupo (OTF- ) y el catión del aminoácido (el grupo $\mathrm{NH}_{3}{ }^{+}$y entre el catión del líquido iónico $\left(\mathrm{BMIm}^{+}\right)$y el anión del aminoácido (COO-). II. lon-hidrofóbica entre los grupos BMlm+OTF- del líquido iónico y los grupos apolares presentes en el aminoácido y entre los grupos $\mathrm{NH}_{3}+/ \mathrm{COO}$ del aminoácido y los grupos alquílicos presentes en el líquido iónico. III. Hidrofóbica-hidrofóbica entre los grupos alquílicos presentes en el líquido iónico y los grupos apolares del aminoácido; El predominio de las interacciones tipo I produce valores positivos de $\Delta V_{\phi}^{o}$; mientras que el predominio de las interacciones tipo II y III da lugar a valores negativos $\Delta V_{\phi}^{o}$; los valores obtenidos en este trabajo son negativos, por tanto pueden ser interpretados como un predominio de las interacciones entre el grupo metil de la DL-alanina con los grupos metil, butil, del líquido iónico [BMIm][OTF], así como de los centros argados de la DL-alanina con los del líquido iónico, el predomino de estas interacciones ocasiona un aumento de las moléculas de agua liberadas desde la esfera de hidratación hasta 
la fase voluminosa produciéndose una disminución en la estructura global del agua, producto del solapamiento de las coesferas. Por otra parte, los valores de $\Delta V_{\phi}^{o}$ disminuyen con el incremento de la concentración de [BMIm][OTF] lo cual indica que aumenta el predominio de las interacciones tipo II.

De acuerdo con (Natarajan et al., 1990;Tome et al., 2009) los $\Delta V_{\phi}^{o}$ para los aminoácidos pueden ser considerados como el resultado de la suma del volumen de Van der Waals $V_{v w}$, el volumen asociado con los huecos o espacios vacios $V_{V}$ y el volumen de contracción debido a la electrostricción $V_{s}$. Asumiendo que $V_{v w}$ y $V_{v}$ tienen la misma magnitud en agua que en soluciones acuosas para la misma clase de solutos ( Palani et al., 2011), los valores de $\Delta \mathrm{V}_{\phi}^{o}$ se pueden explicar de acuerdo al cambio en el volumen de contracción debido al efecto de electrostricción, por tanto la presencia [BMIm] [OTF] aumenta el efecto de electrostricción causada por el aminoácido, a causa de los grupos metil y butil del [BMlm][OTF], lo cual resulta en un incremento de la contracción. Este efecto se debe reflejar en los valores de número de hidratación calculados utilizando el tratamiento de (Millero et al., 1978), según el cual el volumen molar aparente a dilución infinita se puede representar mediante la ecuación (6):

$\bar{V}_{2}^{0}=\bar{V}_{2 \text { int }}^{0}+\bar{V}_{2 \text { elect }}^{0}$

Donde $\bar{V}_{2 \text { int }}^{0}$ es el volumen molar parcial intrínseco del aminoácido, el cual se puede expresar como la adición del volumen de Van der Wals y el volumen debido al efecto de empaquetamiento y $\bar{V}_{2 \text { elect }}^{0}$ es el volumen de electrostricción. Los valores de $\bar{V}_{2 \text { int }}^{0}$ para los aminoácidos pueden ser obtenidos del volumen molar del cristal utilizando la ecuación (7) (Singh et al., 2007):

$\bar{V}_{2 \text { int }}^{0}=\left(\frac{0,7}{0,634}\right) \bar{V}_{\text {cristal }}^{0}$

Donde $\overline{\mathrm{V}}_{\text {cristal }}^{0}$ es el volumen del cristal y se obtiene dividiendo el peso molecular del cristal por su densidad. Tanto la densidad del sólido y el volumen del cristal se asumen constantes en todo el rango de temperatura de trabajo. Pese a que Millero utilizó esta ecuación para aminoácidos en agua, esta ha sido utilizada también en solventes acuosos mixtos (Natarajan et al., 1990; Tome et al., 2009., Liu, 2010 y Singh et al., 2007). Conociendo el volumen de electrostricción es posible determinar el número de moléculas hidratadas alrededor del aminoácido o número de hidratación utilizando la siguiente ecuación:

$\bar{V}_{2 \text { elect }}^{0}=N_{H}\left(\bar{V}_{E}^{0}-\bar{V}_{B}^{0}\right)$

donde $\bar{V}_{\mathrm{E}}^{0}$ es el volumen molar del agua en la esfera de hidratación y $\overline{\mathrm{V}}_{\mathrm{B}}^{0}$ es el volumen molar del agua natural. Este modelo asume que por cada molécula de agua que pase desde la fase voluminosa hasta la región cercana al aminoácido el volumen decrece en $\left(\bar{V}_{\mathrm{E}}^{0}-\overline{\mathrm{V}}_{\mathrm{B}}^{0}\right)$ Usando los valores de $-2.9,-3.3,-4.0 \mathrm{~cm}^{3}$ $\mathrm{mol}^{-1}$ a 288.15, 298.15 y $308.15 \mathrm{~K}$ respectivamente reportados por (Yan et al., 2004) para la relación $\left(\overline{\mathrm{V}}_{\mathrm{E}}^{0}-\overline{\mathrm{V}}_{\mathrm{B}}^{0}\right)$ y obteniendo los valores a 283.15, 293.15, 303.15 y $313.15 \mathrm{~K}$ mediante la correlación de los datos obtenidos por (Yan et al., 2004)se obtienen los valores de $-2.7,-3.1,-3.6$ y $-4.6 \mathrm{~cm}^{3} \mathrm{~mol}^{-1}$ a las temperaturas de $288.15,298.15$ y $308.15 \mathrm{~K}$ respectivamente. Por tanto, los números de hidratación pueden ser obtenidos como una aproximación utilizando la ecuación (8) y los valores se muestran en la Tabla 6.

Tabla 6: Número de hidratación $\mathrm{N}_{H}$ para el aminoácido DL- alanina, en soluciones acuosas de [BMIm][OTF] a las temperaturas de 283.15, 288.15, 293.15, 298.15, 303.15, 308.15, 313.15 K y $318.15 \mathrm{~K}$.

\begin{tabular}{ccccccccc}
\hline \multirow{2}{*}{$\mathrm{T}(\mathrm{K})$} & \multicolumn{8}{c}{$m[\mathrm{BMIm}][\mathrm{OTF}]\left(\mathrm{mol}_{\mathrm{Kg}}{ }^{-1}\right)$} \\
\cline { 2 - 9 } & 0.0100 & 0.0300 & 0.0500 & 0.0800 & 0.2000 & 0.4000 & 0.8000 & 1.0000 \\
\hline 283.15 & 5.9 & 5.9 & 6.0 & 6.0 & 6.0 & 6.1 & 6.2 & 6.2 \\
288.15 & 5.4 & 5.4 & 5.5 & 5.5 & 5.5 & 5.6 & 5.6 & 5.7 \\
293.15 & 5.0 & 5.1 & 5.1 & 5.1 & 5.2 & 5.2 & 5.2 & 5.2 \\
298.15 & 4.7 & 4.7 & 4.7 & 4.8 & 4.8 & 4.8 & 4.9 & 4.9 \\
303.15 & 4.3 & 4.3 & 4.3 & 4.4 & 4.4 & 4.4 & 4.4 & 4.5 \\
308.15 & 3.8 & 3.8 & 3.9 & 3.9 & 3.9 & 4.0 & 4.0 & 4.0 \\
313.15 & 3.3 & 3.4 & 3.4 & 3.4 & 3.4 & 3.5 & 3.5 & 5.6 \\
\hline
\end{tabular}


En la Tabla 6, se observa que los valores de $\mathrm{N}_{\mathrm{H}}$ encontrados para la DL-alanina en soluciones acuosas de [BMIm][OTF] son positivos, los cuales disminuyen conforme aumenta la temperatura y aumentan ligeramente con el incremento en la concentración del [BMIm][OTF] en el solvente. Estos resultados son similares a los encontrados previamente para la mezcla seudobinaria de la Glicina en soluciones acuosas de tetrafluoroborato de 1-butil, 3-metilimidazolio (Páez et al., 2014).

\section{CONCLUSIONES}

En este estudio, se obtuvieron datos volumétricos para la DL-alanina en soluciones acuosas de [BMIm][OTF] a diferentes concentraciones. La segunda derivada de $V_{\phi}^{0}$ con respecto a la temperatura muestra que la DL-alanina se comporta como un soluto desestabilizador de la estructura del solvente en soluciones acuosas de [BMIm][OTF]. Los volúmenes molares aparentes de transferencia a dilución infinita sugieren una disminución de la estructura del agua alrededor de los grupos hidrofóbicos e hidrofílicos. Además, los resultados de los números de hidratación $\mathrm{N}_{\mathrm{H}}$ utilizando el método de Millero, sugieren una disminución en el efecto de electrostricción a medida que se incrementan la temperatura. Es evidente que los parámetros previamente citados contribuyen al entendimiento del ambiente molecular en el que se encuentran inmersas las distintas especies en solución.

\section{AGRADECIMIENTOS}

Los autores agradecen a la Universidad de Córdoba por el apoyo prestado para la realización de este trabajo.

\section{REFERENCIAS}

Armarego, W. C., Purification of laboratory chemicals, $3^{a}$ edición, Heinemann, B, United states of America, (2009).

Cortés, E., A. Dondero., H. Aros y C. Carlesi, Síntesis del líquido iónico bmin ${ }^{+} \mathrm{HSO}_{4}-$ mediante una sola etapa, para aplicaciones en hidrometalurgia, Inf. Tecnol: 21(3), 67-76 (2010).

Deeying, N., Sagarik. K., Effects of metal ion and solute conformation change on hydration of small amino acid, Biophys. Chem: 125, 72-91 (2009).

Hepler, I., Thermal expansion and structure in water and aqueous solutions, Can J. Chem. 47, 4613-4617 (1969).

Liu, C., Volumetric properties of amino acids in aqueous $n$-methylacetamide solutions at $298.15 \mathrm{~K}$, J solution. chem: 39, 1253-1263 (2010).

Makhatadze, G. I., P. L. Privalov, Protein interactións with urea and guanidium chloride: A calorimetric study, J. Mol. Biol: 226, 491-505 (1992).

Millero, F. J., A. Lo surdo y C.Shin, The apparent molal volumes and adiabatic compressibilities of aqueous amino acids at 25. degree, C. J. phys. chem: 82 (7), 784-792 (1978).

Natarajan, M., R. K. Wadi y H. C. Gaur, Apparent molar volumes and viscosities of some alpha and alpha omega amino acids in aqueous ammonium chloride solutions at 298.15 K, J. Chem. Eng. Data: 35 (1), 87-93 (1990).

Páez, M., A. Alvis y G. Arrázola, Efecto de la temperatura sobre la viscosidad de soluciones acuosas diluidas de Cloruro de 1-Butil-3-metil imidazolio [Bmim+ ][Cl], Inf. Tecnol: 25(3), en prensa (2014).

Páez, F., M. Páez y A. Portacio, .Interacciones de la glicina en soluciones acuosas de tetrafluoroborato de 1butil, 3-metilimidazolio a diferentes temperaturas, Quim. Nova: 37(3), 418-425 (2014).

Páez, M., M. Vergara., y E. Montes, Parámetros de interacción ión volumétricos del sistema 1-Butil-3-metil imidazolio trifluorometanosulfonato + agua a varias temperaturas, Inf. Tecnol: 24(6), 47-58 (2013).

Páez, M., P.Cantero y J. Marzola, Densidades y propiedades volumétricas de la glicina en soluciones acuosas de tiosulfato de sodio pentahidratado $\left(\mathrm{Na}_{2} \mathrm{~S}_{2} \mathrm{O}_{3} .5 \mathrm{H}_{2} \mathrm{O}\right)$ a diferentes temperaturas, Rev. Colomb. Quim:41(3), 449-484 (2013).

Pal, A. y N. Chauhan, Volumetric behaviour of amino acids and their group contributions in aqueous lactose solutions at different temperatures, J. Chem. Thermodyn: 43, 140-146 (2011). 
Palani, R., S. Balakrishnan y G. Arumugam, Ultrasonic studies of amino acids in aqueous sucrose solution at different temperatures, phys. sci: 22 (1), 131-141 (2011).

Sharp, K. A., B. Honig, Salt effects on nucleic acids, Curr. Opin. Struct. Biol: 5, 323-328 (1995).

Shekaari, $\mathrm{H}$ y F. Jebali, Densities and electrical conductances of amino acids + ionic liquid $([\mathrm{Hmim}] \mathrm{Br})+\mathrm{H}_{2} \mathrm{O}$ mixtures at 298.15K, Fluid. Phase. Equilib: 295, 68-75 (2010).

Singh, M., M. Pandey., R. Yadav y K.H. Verma, Thermodynamic studies of molar volume, pair and triplet interactions at increasing side-chain length of [alpha]-amino acids in aqueous potassium chloride solutions at different concentration and 310.15 K, J. mol. liq: 135, 188-191 (2007).

Singh, T., A. Kumar, Physical and excess properties of a room temperature iónic liquid (1-methyl-3octylimidazolium tetrafluoroborate) with $n$-alkoxyethanols (C1Em, $m=1$ to 3 ) at $T=(298,15$ to 318,15$) \mathrm{K}$, J, Chem. Thermodyn: 40, 417-423 (2008).

Tome, L. I. N., M. Domınguez y otros cuatro autores: On the Interactions between amino acids and lonic liquids in a media, J. Phys. Chem. B: 113, 13971-13979 (2009).

Yan, Z., J. Wang., W. Kong y Lu. J, Effect of temperature on volumetric and viscosity properties of some [alpha]-amino acids in aqueous calcium chloride solutions, fluid. phase equilib: 215 (2), 143-150 (2004) 